\title{
Published paper
}

M. Oliva-Ramírez, V.J. Rico, J. Gil-Rostra, O. Arteaga, E. Bertran, R. Serna, A.R. González-Elipe, F. Yubero, Applied Surface Science 475, 230-236 (2019)

DOI: https://doi.org/10.1016/j.apsusc.2018.12.200

\section{Liquid switchable radial polarization converters made of sculptured thin}

\section{films}

Manuel Oliva-Ramirez ${ }^{1}$, Victor J. Rico ${ }^{1}$, Jorge Gil-Rostra ${ }^{1}$, Oriol Arteaga ${ }^{2}$,Enric Bertran ${ }^{2}$, Rosalía Serna ${ }^{3}$, Agustín R. González-Elipe ${ }^{1}$, Francisco Yubero ${ }^{1 *}$

${ }^{1}$ ICMSe (CSIC - Univ. Seville), CICCartuja, C/ Américo Vespucio 49, E-41092 Seville, Spain

${ }^{2}$ ENPHOCAMAT (FEMAN) Group, Department of Applied Physics, Universitat de Barcelona, Marti i Franques 1, E-08028, Barcelona, Catalonia, Spain Institute of Nanoscience and Nanotechnology (IN2UB), Universitat de Barcelona, Spain

${ }^{3}$ Institute of Optics "Daza de Valdés" C/ Serrano, 121, E-28006 Madrid, Spain.

*Corresponding author email: yubero@icmse.csic.es

\section{Abstract}


A radial polarization converter is a super-structured optical retarder that converts a conventional linearly polarized light beam into a structured beam with radial or azimuthal polarization. We present a new type of these sophisticated optical elements, which is made of porous nanostructured sculptured single thin films or multilayers prepared by physical vapor deposition at an oblique angle. They are bestowed with an axisymmetric retardation activity (with the fast axis in a radial configuration). In particular, a Bragg microcavity multilayer that exhibits a tunable transmission peak in the visible range with a retardance of up to $0.35 \mathrm{rad}$ has been fabricated using this methodology. Owing to the highly porous structure of this type of thin films and multilayers, their retardance could be switched off by liquid infiltration. These results prove the possibility of developing wavelength dependent (through multilayer optical design) and switchable (through vapor condensation or liquid infiltration within the pore structure) radial polarization converters by means of oblique angle physical vapor deposition.

Keywords: q-plates, radial polarization converters, oblique angle deposition, sculptured thin films

\section{Graphical abstract}




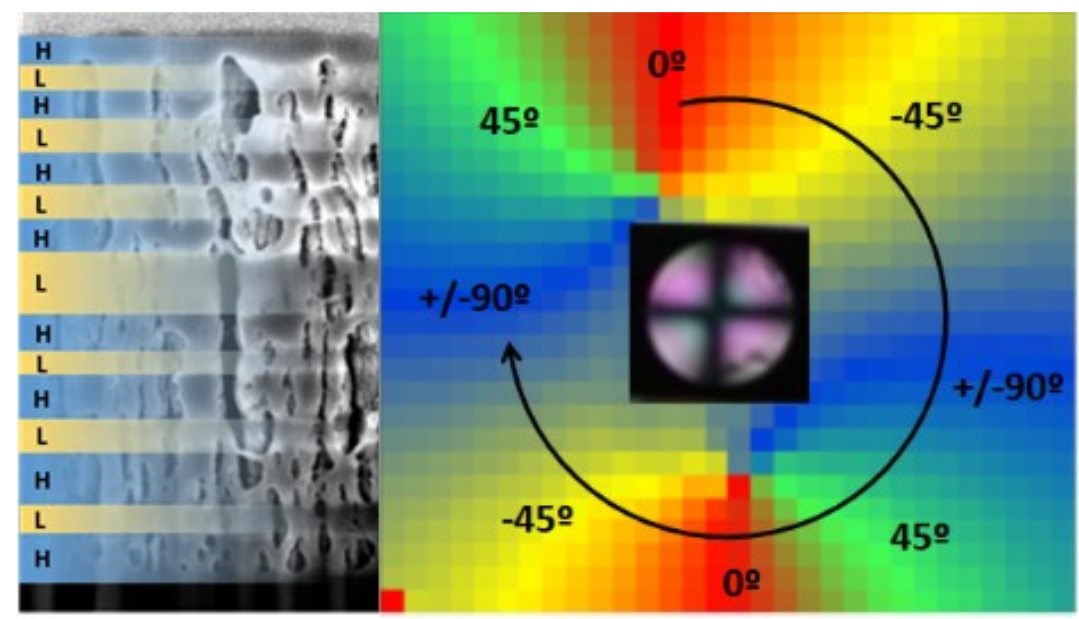




\section{Highlights}

- Porous nanostructured sculptured multilayer with axisymmetric optical activity

- Wavelength dependent response through multilayer optical design

- Optical activity switchable through liquid infiltration within the pore structure

- To be considered as thin film radial polarization converters 


\section{Introduction}

Sculptured thin films prepared by electron beam evaporation in an oblique angle deposition (OAD) geometry have been proposed as optically active components in photonic devices [14]. By this procedure, fancy anisotropic architectures formed by columnar nanostructures with slanted, zig-zag, S-shape or helix arrangements can be achieved varying the orientation of the incoming flux of material with respect to the sample surface (e.g., through the azimuthal rotation of sample during deposition) [1]. Optical birefringence can be obtained with a bundled arrangement of nanocolumns that depicts a thin film microstructural anisotropy that can be tailored by controlling the polar and azimuthal deposition angles [5-7]. These outstanding optical properties have prompted the incorporation of this type of thin films into optically active devices such as retarders [8], tunable circular polarizers [9] lasers with tuned polarization emission [10], mirrors for circular polarized light [11-12], microfluidics liquid detection $[6,13]$, or vapor sensing $[7,14]$.

In the present paper, we propose the synthesis and characterization of $2 \mathrm{D}$ axisymmetric porous birefringent $\mathrm{OAD}$ thin films and multilayers that behave as radial polarization converters (also known as q-plates), a type of super-structured optical retarders where the principal optical axes rotates axially about the center of the optical element [15]. This feature confers these plates the ability to generate light beams with orbital momentum, usually called light cylindrical vector beams $(\mathrm{CV})$, that are characterized by axial symmetry both in amplitude and phase, as well as a rotational symmetry distribution of polarization states. The enhanced focusing properties of $\mathrm{CV}$ beams are of particular interest for 3D focus engineering, optical trapping and laser machining applications [16].

Several methods are reported to generate $\mathrm{CV}$ beams. They can be classified into active methods, involving the direct generation of the beam from a laser cavity [17,18], and passive methods involving optical devices that modify the polarization state of a polarized light beam [19-23]. These passive methods rely on specially designed polarization-state converters that 
commonly involve complex optical elements, such as a liquid-crystal converters $[19,20]$, photo-aligned liquid crystal polymers [21], space-variant subwavelength gratings [22], or a spatially variable segmented half-waveplate [23] that converts light beams with spatially homogeneous polarizations into polarized vortices. Another typical example of this type of passive optical devices is an array of waveplates arranged as slices of a pie chart (eight, for example) where the optical principal axis of each slice is directed towards the center of the optical element [23]. In the present paper, we have developed a one-step method to fabricate radial polarization converters based on porous $\mathrm{OAD}$ thin films and multilayers. Besides analyzing the optical performance of the synthesized polarization converters, we prove the switchability of their retardance by means of the infiltration of a liquid within their open porous structure. Optofluidic switching of the optical response, a completely different approach than the typical electrical switching of this kind of devices [24], is expected to open new alternatives for the development of polarization converters with a big impact in microfluidic and similar applications.

\section{Materials and methods}

Single layer and multilayer porous nanostructured films bestowed with axisymmetric optical activity where deposited on either fused silica plates or polished Si wafer substrates by electron beam evaporation of either $\mathrm{TiO}$ or $\mathrm{SiO}_{2}$ pellets at a process pressure of $3 \times 10^{-4}$ mbar. An $\mathrm{O}_{2}$ inlet was let into the preparation chamber to get fully oxidized, and therefore transparent, $\mathrm{TiO}_{2}$ or $\mathrm{SiO}_{2}$ thin films. The base pressure of the vacuum vessel was $1 \times 10^{-6} \mathrm{mbar}$ and the deposition rate was about $0.1 \mathrm{~nm} / \mathrm{s}$. The angle of incidence of the vapor flux was $\sim 75^{\circ}$ and the crucible-sample distance was about $430 \mathrm{~mm}$. Azimuthal sample rotation (about 30 turns per minute with rotation axis about the center of the sample) was used to achieve an axisymmetric 2D microstructural arrangement. Figure 1 shows schemes of the top (left), front (center) and side (right) views of the particular geometrical oblique angle deposition 
arrangement used to synthesize radial planar converters. Basically, this experimental device consisted of a slit mask $(\sim 15 \mathrm{~mm}$ size made of a $0.5 \mathrm{~mm}$ thick metal foil with a $2 \mathrm{~mm}$ perpendicular slit) placed $\sim 30 \mathrm{~mm}$ from the sample location in the line of sight between substrate and crucible. The particular location and dimensions of the slit impose a partial collimation to the flux of particles that, as described in the results section, renders thickness homogeneity to the coatings while bestowing them some degree of birefringence.

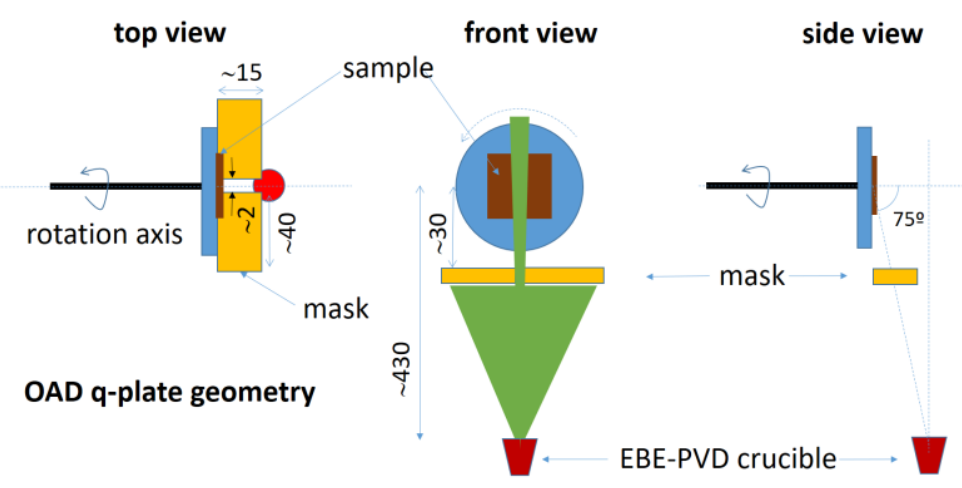

Figure 1. Top view (left), front view (center), and side view (right) schemes of the geometrical oblique angle deposition arrangement (dimensions are not at scale).

Three types of samples (size $25 \times 25 \mathrm{~mm}$ ) were prepared: i) single layer $\mathrm{TiO}_{2}$ porous films (qTiO2) about $600 \mathrm{~nm}$ thick, ii) single layer $\mathrm{SiO}_{2}$ porous films (qSiO2) about $600 \mathrm{~nm}$ thick, and iii) a microcavity (qBM) formed by 2 porous Bragg mirrors of 7 layers with a stacked HLHLHLH structure $\left(\mathrm{H}\right.$ and $\mathrm{L}$ refer to $\mathrm{TiO}_{2}$ and $\mathrm{SiO}_{2}$ films with high and low refractive indices, respectively) and a thicker $\mathrm{SiO}_{2}$ defect layer in between. The microcavity was designed to depict a transmission peak at about $550 \mathrm{~nm}$ in the middle of the reflection band of the individual Bragg mirrors. Typical thickness of individual $\mathrm{TiO}_{2}$ and $\mathrm{SiO}_{2}$ layers of the Bragg mirrors was $\sim 80 \mathrm{~nm}$, while the central $\mathrm{SiO}_{2}$ layer acting as optical defect had a thickness of $\sim 180 \mathrm{~nm}$. 
Samples were characterized by SEM, transmission UV-Vis, mapping spectroscopic ellipsometry (SE) and spatially resolved Muller Matrix polarimetry.

The microstructure of the $\mathrm{qTiO} 2$ and $\mathrm{qSiO} 2$ samples deposited on polished $\mathrm{Si}(100)$ wafers (cross sectional and planar views) was characterized by scanning electron microscopy (SEM) using a Hitachi S4800 field emission microscope. The qBM sample was also characterized by FIB-SEM using a procedure similar to that described in ref.[6].

UV-Visible transmission spectra of the qBM samples deposited on fused silica were recorded in a Varian Cary100 spectrophotometer.

Lateral thickness distribution and birefringence across the $\mathrm{SiO}_{2}$ and $\mathrm{TiO}_{2}$ samples surface were characterized by spectroscopic ellipsometry using a Woollan VASE apparatus provided with an accessory for spatial mapping detection. The size of the analysis probe was about 1 $\mathrm{mm}^{2}$. Measurements were taken along an orthogonal grid of $2 \mathrm{~mm}$ pitch. The $\Psi$ and $\Delta$ ellipsometric parameters were determined using a biaxial model for the individual single material anisotropic layers. Basically, the model consists of an orthorhombic optical description of the deposited films, with a main axis perpendicular to the sample surface and the other two axis at orthogonal directions parallel to the sample surface with Euler angles defined as THETA $=0^{\circ}, \mathrm{PSI}=0^{\circ}$ and $\mathrm{PHI}$ as free fitting parameter. PHI represents the in-plane orientation of the optical axis at the sample surface plane. The mathematical description of the refractive index for each direction was done by an effective medium approximation model (isotropic Bruggeman) that considered the mixture of either $\mathrm{SiO}_{2}$ or $\mathrm{TiO}_{2}$ compact materials and voids. For the compact materials, a Cauchy type wavelength dispersion was used to describe the dependence of the refractive index with wavelength. Percentage of voids along each direction was taken as free parameter of the spectroscopic ellipsometric analysis. The main aim of this description is to get a good and independent account of the refractive index along every direction (in the sense of being able to fit the spectroscopic ellipsometry data) 
and, at the same time, to get a direct estimation of the "empty" space in each direction (according to the effective medium Bruggeman parametrization).

The optical activity of the samples was also characterized by means of spatially resolved transmission measurements in a Mueller matrix (MM) polarimeter. This apparatus, described in detail in ref.[27], measures simultaneously all elements of the $4 \times 4$ Mueller matrix thanks to four different photoelastic modulators. The sample was mounted in a two-axis motorized sample holder that allows generating 2D Mueller matrix maps. These maps, which are presented as an image, represent an area of $15 \times 13 \mathrm{~mm}^{2}$ that is sampled in steps of $0.5 \mathrm{~mm}$ and entails 896 measurements at every selected wavelength. The diameter of the probing beam was $0.8 \mathrm{~mm}$. The transmission Mueller-matrix measured at each point was processed to calculate, among others, the linear birefringence (LB) defined here as $2 \pi$ times the retardance in waves [26]. It is noteworthy that the optical activity is not intrinsic of the involved materials, but arises from the special microstructure of the samples.

\section{Results}

\subsection{Film structure}

Three types of samples (size $25 \times 25 \mathrm{~mm}$ ) were prepared by electron beam evaporation at oblique geometry using a special geometrical experimental set-up (see experimental section for details): i) single layer $\mathrm{TiO}_{2}$ porous films (qTiO2), ii) single layer $\mathrm{SiO}_{2}$ porous films (qSiO2), and iii) a Bragg microcavity (qBM), formed by 2 porous Bragg mirrors and a thicker $\mathrm{SiO}_{2}$ defect layer in between, and that was designed to depict a transmission peak at about 550 $\mathrm{nm}$ in the middle of the reflection band of the individual Bragg mirrors.

The deposited single films have a porous vertical columnar microstructure as shown in the SEM crossed section micrographs included in Figure S1 (supporting information) corresponding to the $\mathrm{qTiO} 2$ (left) and $\mathrm{qSiO} 2$ (right) samples. Development of vertical 
nanocolumns in OAD thin films results from the azimuthal rotation of the substrate during the deposition process [1,2]. No significant difference in microstructure was observed for cross section micrographs taken along different directions or at different sample location. The section of the individual columns was about $\sim 60 \mathrm{~nm}$ for the $\mathrm{qSiO} 2$ sample and slightly smaller for the qTiO2 sample (see size of the nano-column tips in the front view SEM images of samples).

A similar microstructure (vertical nanocolumns and similar width) was obtained for the qBM sample. Figure 2 shows a typical FIB-SEM image of the multilayered ( $\left.\mathrm{H}: \mathrm{TiO}_{2}, \mathrm{~L}: \mathrm{SiO}_{2}\right)$ structure of this sample. It consists of vertical nanocolumns laterally associated at some points independently of layer material and vertical void spaces distributed all over the sample thickness. Filling this pore space with liquids is the basis of the optofluidic switching of the polarization activity and will be presented at the end of this paper.

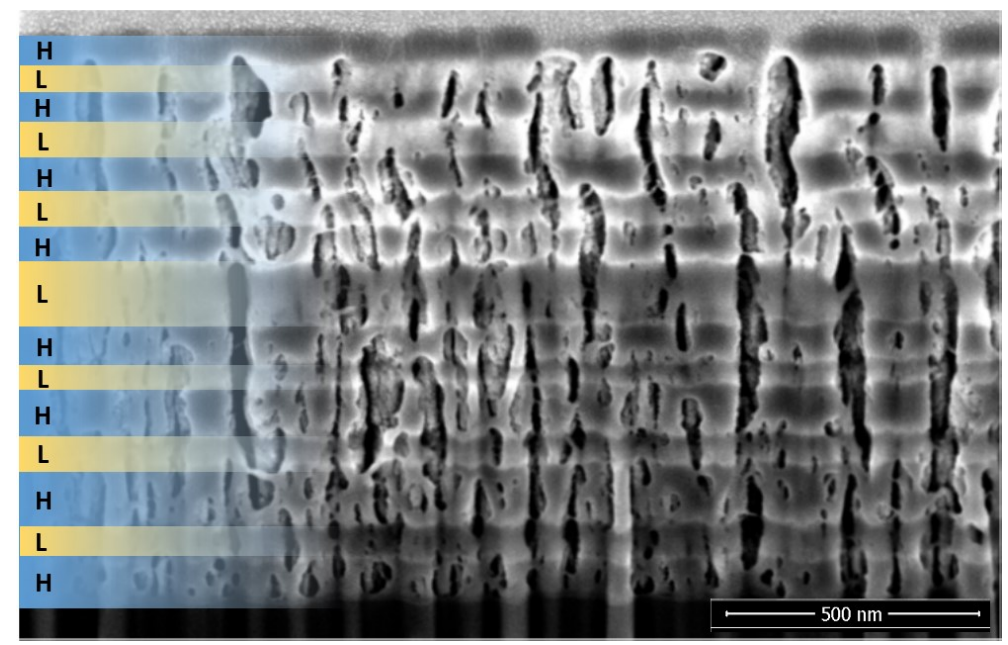

Figure 2. FiB-SEM of qBM sample; $L$ and $\mathrm{H}$ refer to $\mathrm{SiO}_{2}$ and $\mathrm{TiO}_{2}$ porous materials within the superimposed layered scheme. 
Mapping spectroscopic ellipsometry (SE) analysis of the qTiO2 sample is reported in Figure 3. The thickness mapping in Figure 3-top reveals a small 4\% gradation in thickness from the center to $5 \mathrm{~mm}$ away from it. This rather constant distribution of thickness proves that the selected geometry and deposition parameters ensure thickness homogeneity while keeping some in-plane birefringence as evidenced by the SE analysis in Figure 3-bottom. This figure shows the in-plane birefringence $2 \mathrm{D}$ profile map of the same sample. It was obtained by leastsquared fitting of the SE signal at different points of the sample. This position dependent SE analysis indicates that the strength of the in-plane birefringence $n_{b}-n_{p b}$ (where $n_{b}$ is the refractive index in the direction tangent to circumferences around the center of the sample and $n_{p b}$ the refractive index along the radius of these circumferences) increases from zero (i.e., no in-plane optical anisotropy) at the sample center to about 0.012 RIU (refractive index units) at about $6 \mathrm{~mm}$ away from the center (i.e., the increase of birefringence $\Delta\left(n_{b}-n_{p b}\right)$ is about 0.002 RIU/mm). This analysis also indicates that the direction of the in-plane optical axis rotates azimuthally according to an axisymmetric distribution around the center of the sample. This demonstrates that the $\mathrm{qTiO} 2$ sample presents $2 \mathrm{D}$ axisymmetric birefringence. Similar results were obtained for the $\mathrm{qSiO} 2$ sample, although the magnitude of the in-plane birefringence was about half the one of qTiO2, i.e., $n_{b}-n_{\perp b}=0.006$ RIU at $6 \mathrm{~mm}$ from the center (i.e., $\Delta\left(n_{b^{-}}\right.$ $\left.\left.n_{p b}\right) \cong 0.001 \mathrm{RIU} / \mathrm{mm}\right)$. 

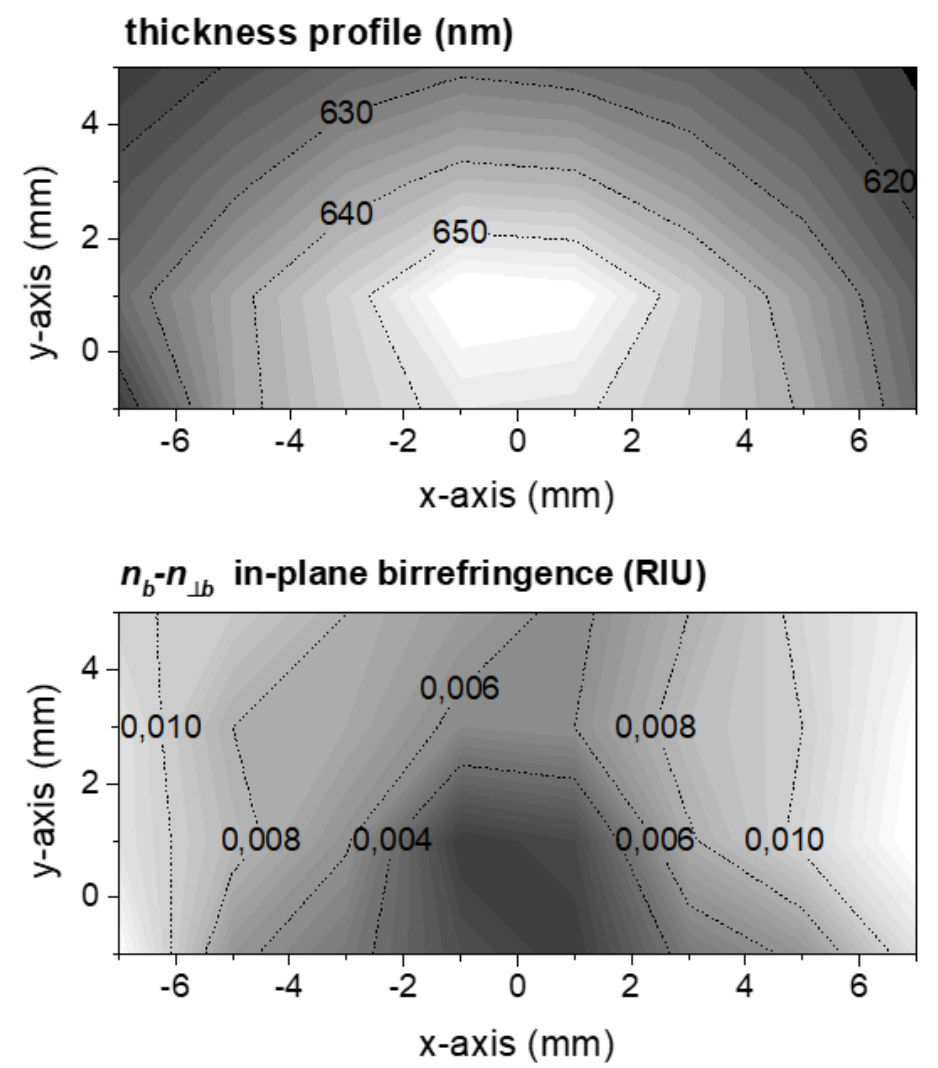

Figure 3. Thickness (top) and $\mathrm{n}_{\mathrm{b}} \mathrm{n}_{\mathrm{pb}}$ in-plane birefringence (bottom) 2 D profiles of qTiO2 sample obtained by mapping SE analysis

\subsection{Axisymmetric optical activity of the qBM multilayer sample}

A means to make the observed super-structured axisymmetric birefringent samples wavelength dependent is to fabricate a Bragg microcavity by the stacking of $\mathrm{qSiO} 2$ and qTiO2 single layers according to the multilayer architecture described in the experimental section. The characteristic optical response of such an optical system consists of a transmission peak opened in the reflection band of the two Bragg mirrors forming the microcavity [6].

For the qBM sample prepared on a glass plate, a direct assessment of the spatial distribution of the anisotropic optical response was obtained by measuring its transmittance between 
either aligned $\left(\mathrm{T}_{\mathrm{uu}}\right)$ or crossed $\left(\mathrm{T}_{\mathrm{ux}}\right)$ polarizers at several positions along a line crossing its center. The series of measured $\mathrm{T}_{\text {uu }}$ spectra (included in supporting information Figure S2-left) shows that the transmission peak blue-shifts from $570 \mathrm{~nm}$ at the center of the sample to 530 $\mathrm{nm}$ at $20 \mathrm{~mm}$ away from the sample center. Just at the center, the $\mathrm{T}_{\mathrm{ux}}$ signal vanished (Figure S2-right), indicating lack of optical activity at this position. Meanwhile, at $20 \mathrm{~mm}$ away from the center, a certain $\mathrm{T}_{\mathrm{ux}}$ intensity (i.e., ca. 3\%) was measured at the transmission peak wavelength (c.f., Figure S1-bottom). This simple analysis confirms that a), the optical thickness of the qBM sample slightly decreases from the sample center to the borders (evidenced by the blue shift in $\mathrm{Tuu}_{\mathrm{uu}}$, b) the qBM sample is not optically active at the center (the $\mathrm{T}_{\mathrm{ux}}$ intensity vanishes) and c) the retardance of the sample rises radially from its center to the borders.

Another outstanding feature is that sample qBM presents axisymmetric optical activity. To visualize the spatial distribution of optical activity in this sample we carried out a simple experiment with the experimental set-up schematized in Figure 4-top. The qBM sample deposited on a polished $\mathrm{Si}$ wafer was illuminated at $45^{\circ}$ incidence angle with white light linearly polarized with the polarization vector parallel to the sample surface. Specular reflection at a similar angle was measured after a second polarizer whose transmission axis was initially oriented crossed/orthogonal to the polarization axis of the incident light as shown in the scheme. Figure 4-bottom shows the images recorded as a function of the angular misalignment between the crossed polarizers. It is apparent that when the two polarizers are orthogonally crossed $\left(0.0^{\circ}\right.$ picture $)$, a dark cross zone is observed where light is not transmitted to the detector (i.e., zero intensity). When imposing a certain angular misalignment to the second polarizer some light starts to be transmitted and the extinction cross progressively vanishes with the misalignment angle. This behavior was independent of the azimuthal orientation of the sample and demonstrates a spatially heterogeneous retarder 
effect which is consistent with an axisymmetric rotation of the optical axis of the sample. The pictures in Figure 3-bottom correspond to snapshots of Video V1 included as supporting information.
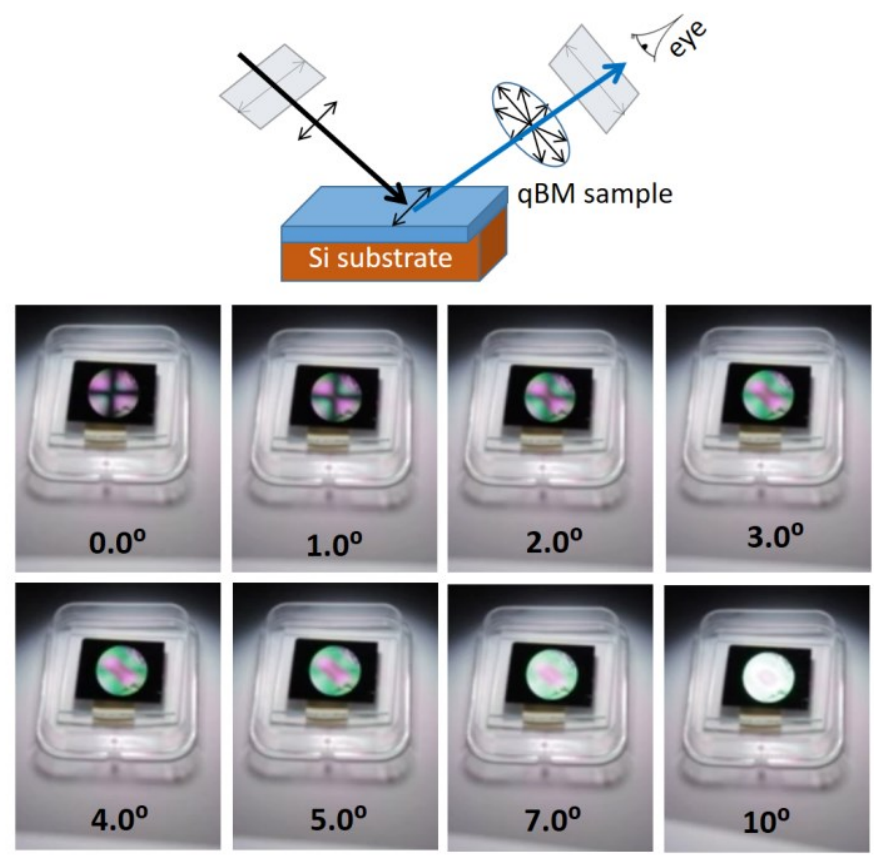

Figure 4. Top) Scheme of the experimental arrangement. Bottom) White light reflected onto the qBM sample after slight misalignment between crossed polarizers (misalignment angle indicated in the figures).

A first attempt to quantify the birefringent behavior of qBM sample was done by SE. However, unlike the analysis carried out with the single qTiO2 and $\mathrm{qSiO} 2$ single layer samples, quantification of the spectroscopic ellipsometric data was not so straightforward here because fitting of data for this complex multilayer system requires an overwhelming number of variables resulting from the 15 layers and 2 biaxial porous materials $\left(\mathrm{TiO}_{2}\right.$ and $\left.\mathrm{SiO}_{2}\right)$ integrated in this structure. Nevertheless, local SE analysis confirmed the consistency of optical characteristics of the individual $\mathrm{TiO}_{2}$ and $\mathrm{SiO}_{2}$ layers stacked in the $\mathrm{BM}$ structure and those for the single qTiO2 and qSiO2 samples described above (c.f. Figure 3). For example, a 
good fitting was obtained for values in the range of 0.08 RIU for the in-plane birefringence of the $\mathrm{TiO}_{2}$ layers stacked in $\mathrm{qBM}$ at a point about $6 \mathrm{~mm}$ away from the center of the sample. This value is significantly larger than those obtained from the SE analysis of the qTiO2 samples, but about half the value previously obtained for BM multilayer architectures with a homogeneous zigzag columnar arrangement [6]. A complete SE fitting analysis at this point off-center of qBM sample is presented in the supporting information, Figure S3.

A more robust quantitative study of the anisotropic birefringent behavior of the qBM sample was achieved by spatially resolved MM polarimetry. Figure 5 shows the raw data corresponding to spatially resolved transmission MM polarimetry measurements at $540 \mathrm{~nm}$ (at the BM transmission peak). Each image represents one of the 16 elements $(\mathrm{m} 00, \ldots, \mathrm{m} 33)$ of the Mueller matrix, for an area of about $15 \times 13 \mathrm{~mm}$ around the center of the sample. Results of the $\mathrm{m} 00, \mathrm{~m} 11, \mathrm{~m} 22$, and $\mathrm{m} 33$ main diagonal and $\mathrm{m} 01, \mathrm{~m} 10, \mathrm{~m} 02, \mathrm{~m} 20, \mathrm{~m} 13, \mathrm{~m} 31, \mathrm{~m} 23$, and m32 off-diagonal terms are shown. A first evidence from these MM maps is the symmetry of the $2 \mathrm{D}$ patterns, a clear indication of the axisymmetric distribution of the optical axis in sample qBM.

In addition to this preliminary assessment, a detailed analysis of the Muller matrix elements was carried out. To help in the interpretation of the measured patterns, Tables S1 and S2 in the supporting information report the expected dependence of the MM elements on the orientation of the optical axis $\varphi$ and the retardance $\alpha$ for the case of a pure linear retarder, and the orientation of the optical axis $\varphi$ and difference in intensity $\delta$ for a pure linear diattenuator [25]. Taken into account these dependences, for a pure homogeneous linear retarder depicting a retardance $\alpha$ and an orientation of the fast axis at an angle $\varphi$, it holds that $\mathrm{m} 23=\cos 2 \varphi$ $* \sin \alpha$, and $\mathrm{m} 13=-\sin 2 \varphi * \sin \alpha$ and $\mathrm{m} 33=\cos \alpha$ [25]. Based on these relations, the pattern of the $\mathrm{m} 13$ and $\mathrm{m} 23$ and $\mathrm{m} 33 \mathrm{MM}$ elements shows that no retardance exists at the center of the 
sample $(\alpha=0, \mathrm{~m} 13=\mathrm{m} 23=0, \mathrm{~m} 33=1)$, independently on the orientation of the optical axes (c.f., see the white color at the center of $\mathrm{m} 13$ and $\mathrm{m} 23$ and red color at the center of $\mathrm{m} 33$ ). Getting away from the sample center, the patterns of the corresponding elements indicate that retardance increases. On the other hand, from the analysis of $\mathrm{m} 23 \mathrm{MM}$ element it can be concluded that the fast axis aligns horizontally at the left/right (maximum positive retardance) and vertically at the top/bottom (maximum negative retardance). Similar arguments may be used to justify the $2 \mathrm{D}$ intensity pattern of the m13 element, now with respect to the $45^{\circ}$ directions

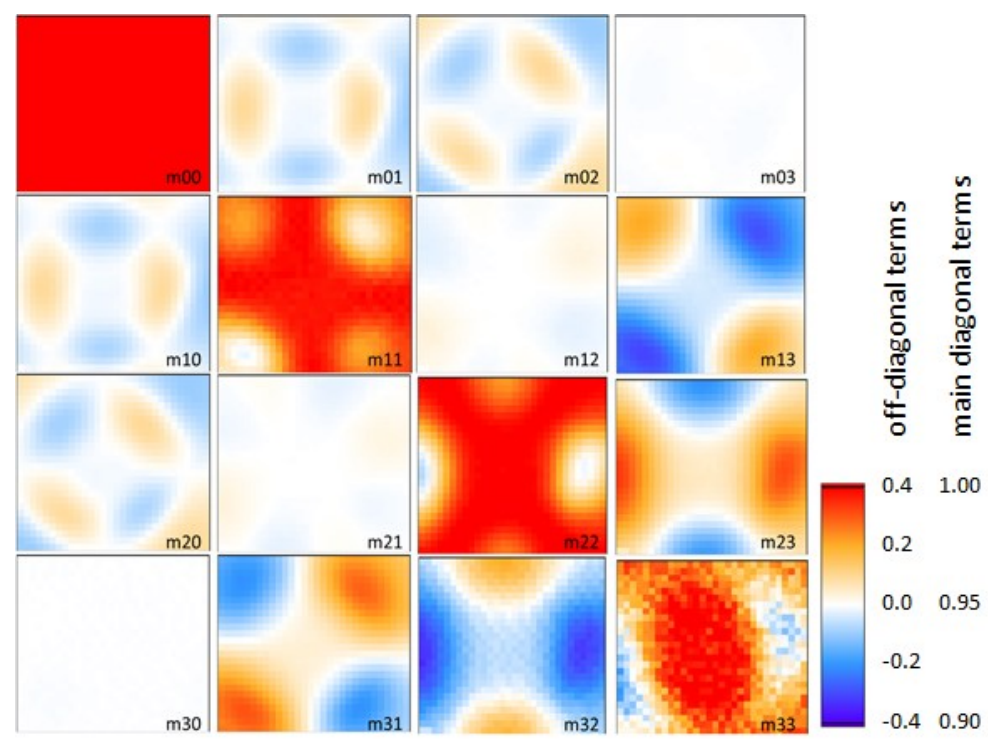

Figure 5. Spatially resolved MM polarimetry measurements evaluated at $540 \mathrm{~nm}$ (i.e., at the transmission peak of the microcavity). The main diagonal MM elements are scaled between 0.9 and 1.0, while the other off-diagonal MM elements are scaled between 0.4 and -0.4.

Meanwhile, the weaker 2D pattern intensity of m01 and m02 MM elements can be accounted for by a linear diattenuator behavior. In this case, it holds that $\mathrm{m} 01=2 \delta^{*} \cos 2 \varphi$ (where $\mathrm{A}$ is proportional to the difference in transmittance between the two orthogonal axes), i.e., m01 follows qualitatively the same pattern distribution than the m23 element. Similarly, under the 
same conditions, $\mathrm{m} 02=2 \delta * \sin 2 \varphi$, i.e., the same pattern shape than that of the $\mathrm{m} 13$ element, but with opposite sign. This indicates that, in addition to the retarder behavior of the qBM, there is also a minor difference in transmission (note the weaker intensity color) between the two optical axes. This effect is due to the different transmittance of the sample as a function of the azimuth angle of the incoming linear polarization.

The qualitative description of the patterns of the off-diagonal terms is also consistent with the patterns observed for the main diagonal $\mathrm{m} 11, \mathrm{~m} 22$ and $\mathrm{m} 33$ elements of the MM.

The evolution of the super-structured retardance of sample qBM can be appreciated more clearly after evaluating the magnitude and orientation of the linear birefringence (LB) with the method described in ref.[26] (c.f. Figure 6). LB magnitude or retardance represents the integrated effect of birefringence acting along the path of a light beam that traverses the sample. If the incident light beam is linearly polarized, the two orthogonal components of the polarized light will exit the sample with a phase difference. Therefore, the maps in this figure show that the magnitude of the in-plane LB increases when moving away from the sample center (Figure 6-left). This effect is consistent with that observed by transmittance measurements between crossed polarizers of the qBM sample and the mapping SE analysis described above for sample qTiO2 (c.f. Figure 4). Furthermore, it is apparent that the spatial distribution of optical axes in sample $\mathrm{qBM}$ is axisymmetric as it is shown in Figure 6-right. Effectively, this figure shows that beyond 3-4 $\mathrm{mm}$ from the sample center a clear smooth change of the orientation of the optical axis takes place: it starts from a horizontal orientation of the fast axis at the top of the sample (c.f., Figure 6-right, red), to turn smoothly clockwise to successive angles of $-45^{\circ}$ (yellow), $-90^{\circ}$ (blue), $45^{\circ}$ (green), and finally to $0^{\circ}$ (red at the bottom of the figure). 

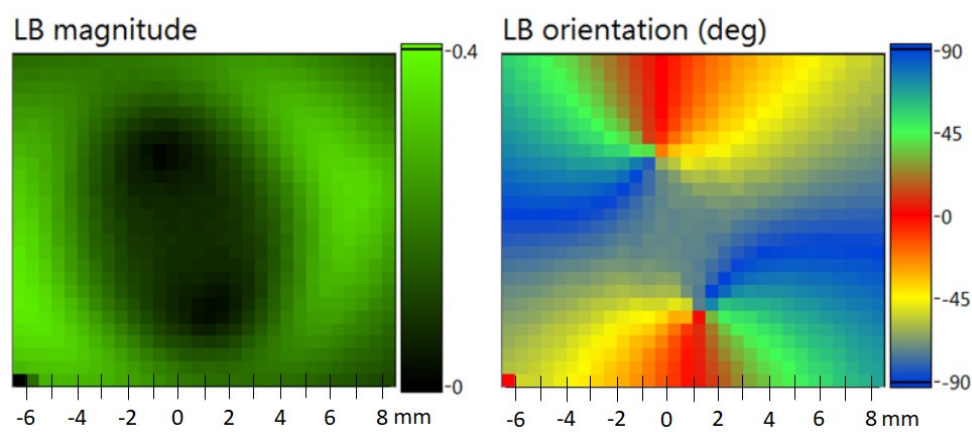

Figure 6. Linear birefringence (LB) magnitude in radians (left) and orientation in degrees (right) maps evaluated at $540 \mathrm{~nm}$ (i.e., at the resonant peak of the microcavity) by the spatially resolved MM polarimetry measurements from Figure 5.

Intensity maps in Figure 5 confirm that the optical principal axis rotate around the center of the sample. This effect takes place not only at the wavelength of the BM transmission peak, but also at other wavelengths (see Figure S4 in supporting information the plots of LB magnitude and orientation at 475 and $650 \mathrm{~nm}$, i.e., off the transmission peak position of the microcavity).

\subsection{Fluidic switching of the radial polarization activity of $q B M$ samples}

The high porosity of the OAD thin films and multilayers (up to $50-60 \%$ of the total volume of the film ${ }^{[6]}$ ) provide a straightforward way for tailoring their optical properties by liquid infiltration. Therefore, the optofluidic behavior of the qBM was characterized after ethanol infiltration in its porous structure. The purpose of this experiment is to check whether the radial polarization activity of the qBM can be switched off by liquid infiltration. Figure 7 shows the variation of the LB magnitude vs wavelength in the qBM sample at a point off the center of the sample before and after water infiltration in its porous structure. A first evidence of the spectra in this figure is that water infiltration of the $\mathrm{qBM}$ produces a decrease in the 
magnitude of the linear birefringence by a factor of ca. 5. This drastic change can be interpreted in the following terms: when the empty pores of the film are filled with ethanol, the optical contrast between different orientations diminished and so does its optical activity. The observed effect was reversible and the LB magnitude recovered its initial value after empting the pores (e.g., drying the BM). Similar optofluidic effects have been reported for vapor and liquid sensing using sculptured slanted or zigzag BM [7].

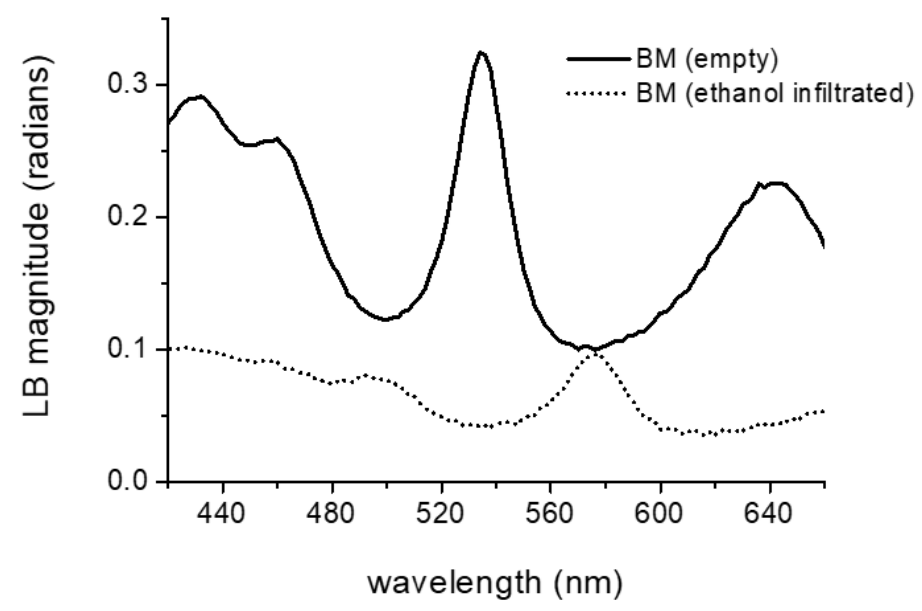

Figure 7. Variation of the LB magnitude vs wavelength at a point off the center of the $q B M$ sample either empty (full line) or ethanol infiltrated (dashed line).

Reversibility of this phenomenon is illustrated in Video V2 included in the supporting information showing that $\mathrm{qBM}$ sample recovers its performance as radial polarization converter after liquid removal (drying in our case).

\section{Conclusions}

The strength of the optical activity of the reported OAD films acting as switchable radial polarization converters ( $0.35 \mathrm{rad}$ or 0.056 waves $)$ is lower than that of standard q-plates with 
$\mathrm{q}=1$ i.e., with an optical axis turned by $360^{\circ}$ around the center of the sample and a phase retardation of PI (i.e., half-wave). Nevertheless, their planar configuration in the form of single thin films or multilayers and the possibility to operate both in transmission and reflection makes the $\mathrm{OAD}$ alternative very interesting for applications where manufacturing simplicity and easy integration with other components are obliged requirements. In this regard the open porosity and, therefore, the demonstrated suitability of these devices for optofluidic operation are of particular interest for microfluidic integration or for their use as responsive devices towards condensed vapors and liquids. It is also of particular interest that the developed technology allows tuning the wavelength response of the device through traditional multilayer optical design. The relatively simple one-step manufacturing procedure by physical vapor deposition constitutes an additional advantage in comparison with the rather complicated procedures of alternative devices.

\section{Acknowledgements}

We acknowledge the support of the European Regional Development Funds program (EUFEDER), the Spanish Ministry Economy, Industry, and Competitiveness (projects 201560E055, MAT2016-79866-R, RECUPERA 2020 and Red CONSOLIDER MAT201569035-REDC). 


\section{Appendix A. Supplementary material}

The following are the Supplementary data to this article: Muller matrix elements of a pure linear retarder and pure linear diattenuator, Cross section and front view SEM micrographs of qTiO2 and qSiO2 samples. Transmittance spectra at normal incidence along a line crossing the center of the qBM sample. Spectroscopic ellipsometric fitting of the qBM multilayer at a point off the center of the sample. Linear birefringence magnitude and orientation maps evaluated at 475 and $650 \mathrm{~nm}$ on $\mathrm{qBM}$ sample by spatially resolved MM polarimetry measurements. Videos of the reflectance of the qBM sample illuminated with white polarized light analyzed with a crossed polarizer and the attenuation of the characteristic extinction cross due to ethanol infiltration within the open porosity of the qBM sample. 


\section{References}

[1] A. Lakhtakia, R. Messier, Sculptured Thin Films, Nanoengineering Morphology and Optics, SPIE Press, 2005.

[2] M.M. Hawkeye, M.T. Taschuk, M.J. Brett, Glancing Angle Deposition of Thin Films: Engineering the Nanoscale, John Wiley \& Sons Ltd, Chichester, UK 2014.

[3] M.W. McCall, I.J. Hodgkinson, Q. Wu, Birefringent Thin Films and Polarizing Elements, Imperial College Press, 2015.

[4] A.R. González-Elipe, A. Barranco, A. Borrás, A. Palmero, Perspectives on oblique angle deposition of thin films: From fundamentals to devices. Progress in Materials Science 76 (2016) 59-153. https://doi.org/10.1016/j.pmatsci.2015.06.003.

[5] H. van Kranenburg, C. Lodder, Tailoring Growth and Local Composition by ObliqueIncidence Deposition: a Review and New Experimental Data Mater. Sci. Eng. 11 (1994) 295354. https://doi.org/10.1016/0927-796X(94)90021-3.

[6] M. Oliva-Ramirez, A. Barranco, M. Loffler, F. Yubero, A.R. González-Elipe, Optofluidic Modulation of Self-Associated Nanostructural Units Forming Planar Bragg Microcavities ACS Nano 10 (2016) 1256-1264. https://doi.org/10.1021/acsnano.5b06625.

[7] M. Oliva-Ramírez, J. Gil-Rostra, M. C. López-Santos, A. R. González-Elipe, F. Yubero, Vapor and Liquid Optical Monitoring with Sculptured Bragg Microcavities J. of Nanophotonics 11 (2017) 046009. https://doi.org/10.1117/1.JNP.11.046009.

[8] R.J. Martin-Palma, F. Zhang, A. Lakhtakia, A. Cheng, J. Xu, C.G. Pantano, Retardance of Chalcogenide Thin Films Grown by the Oblique-Angle-Deposition Technique Thin Solid Films 517 (2009) 5553-5556. https://doi.org/10.1016/j.tsf.2009.03.200. 
[9] K.M. Krause, M.J. Brett, Spatially Graded Nanostructure Chiral Films as Tunable Circular Polarizers Adv. Func. Mat. 18 (2008) 3111-3118. https://doi.org/10.1002/adfm.200800685.

[10] F. Zhang, J. Xu, A. Lakhtakia, T. Zhu, S.M. Pursel, M.W. Horn, Circular Polarization Emission from an External Cavity Diode Laser. Appl. Phys. Lett. 92 (2008) 111109. https://doi.org/10.1063/1.2896306.

[11] P. D. McAtee, A. Lakhtakia, Reflection and Transmission of Obliquely Incident Light by Chiral Sculptured Thin Films Fabricated Using Asymmetric Serial-Bideposition Technique J. Nanophotonics 11 (2017) 043502. https://doi.org/10.1117/1.JNP.11.043502.

[12] I. Hodgkinson, Q-h. Wu, B. Knight, A. Lakhtakia, K. Robbie, Vacuum Deposition of Chiral Sculptured Thin Films with High Optical Activity. Appl. Opt. 39 (2000) 642-649. https://doi.org/10.1364/AO.39.000642.

[13] A. Lakhtakia, M.W. McCall, J.A. Sherwin, Q.H. Wu, I.J. Hodgkinson, Sculptured Thin Films Spectral Holes for Optical Sensing of Fluids..Optics Comm. 194 (2001) 33-46. https://doi.org/10.1016/S0030-4018(01)01225-1.

[14] Y.J. Liu, J.Shi, F. Zhang, H. Liang, J. Xu, A. Lakhtakia, High-Speed Optical Humidity Sensors Based on Chiral Sculptured thin Films. Sensors and Actuators B 156 (2011) 593-598. https://doi.org/10.1016/j.snb.2011.02.003.

[15] L. Marrucci, C. Manzo, D. Paparo, Optical Spin-to-Orbital Angular Momentum Conversion in Inhomogeneous Anisotropic Media. Phys. Rev. Lett. 96 (2006) 163905. https://doi.org/10.1103/PhysRevLett.96.163905.

[16] Q. Zhan, Cylindrical Vector Beams: from Mathematical Concepts to Applications. Advances in Optics and Photonics 1 (2009) 1-57. https://doi.org/10.1364/AOP.1.000001.

[17] Y. Kozawa, S. Sato, Generation of a Radially Polarized Laser Beam by Use of a Conical Brewster Prism.Opt. Lett. 30 (2005) 3063-3065. https://doi.org/10.1364/OL.30.003063. 
[18] K. Yonezawa, Y. Kozawa, S. Satoet, Generation of a Radially Polarized Laser Beam by Use of the Birefringence of a c-cut Nd:YVO4 Crystal Opt. Lett. 31 (2006) 2151-2153. https://doi.org/10.1364/OL.31.002151.

[19] M. Stalder, M. Schadt, Linearly Polarized Light with Axial Symmetry Generated by Liquid-Crystal Polarization Converters Opt. Lett. $21 \quad$ (1996) 1948-1950. https://doi.org/10.1364/OL.21.001948.

[20] J. A. Davis, D.E. McNamara, D.M. Cottrell, T. Soneharaet, Two-Dimensional Polarization Encoding with a Phase-only Liquid-Crystal Spatial Light Modulator Appl. Opt. 39 (2000) 1549-1554. https://doi.org/10.1364/AO.39.001549.

[21] S. C. McEldowney, D.M. Shemo, R.A. Chipman, P.K. Smithet, Creating Vortex Retarders Using Photoaligned Liquid Crystal Polymers. Opt. Lett. 33 (2008) 134-136. https://doi.org/10.1364/OL.33.000134.

[22] Z. Bomzon, V. Kleiner, E. Hasmanet, Formation of Radially and Azimuthally Polarized Light Using Space-Variant Subwavelength Metal Stripe Gratings Appl. Phys. Lett. 79 (2001) 1587-1589. https://doi.org/10.1063/1.1401091.

[23] G. Machavariani, Y. Lumer, I. Moshe, A. Meir, S. Jackelet, Efficient Extracavity Generation of Radially and Azimuthally Polarized Beams Opt. Lett. 32 (2007) 1468-1470. https://doi.org/10.1364/OL.32.001468.

[24] S. Slussarenko, A. Murauski, T. Du, V. Chigrinov, L. Marrucci, E. Santamato, Tunable Liquid Crystal q-plates with Arbitrary Topological Charge. Optics Express 19 (2011) 40854090. https://doi.org/10.1364/OE.19.004085.

[25] R. A. Chipman, Polarimetry, chapter 22 in Handbook of Optics II, M. Bass, editor in chief. McGraw-Hill, New York, 1995. 
[26] O. Arteaga, A. Canillas, Analytic Inversion of the Mueller-Jones Polarization Matrices for Homogeneous $\quad$ Media. Opt. $\quad$ Lett. $35 \quad$ (2010) 559-561. https://doi.org/10.1364/OL.35.000559.

[27] O. Arteaga, J. Freudenthal, B. Wang, B. Kahr, Mueller Matrix Polarimetry with Four Photoelastic Modulators: Theory and Calibration Appl. Opt. 51 (2012) 6805-6817. https://doi.org/10.1364/AO.51.006805. 